\title{
Investigation of skin self-examination and sunscreen use in adolescents in northern Cyprus
}

\author{
Didem Mullaaziz ${ }^{1}$, Aslı Kaptanoğlu², Evren Hınçal ${ }^{3}$ Emel Erdal Çalıkoğlư \\ ${ }^{1}$ Department of Dermatology and Venereology, Faculty of Medicine, Near East University, Nicosia, Cyprus \\ 2Department of Dermatology and Venereology, Faculty of Medicine, Marmara University, Istanbul, Turkey \\ ${ }^{3}$ Department of Mathematics, Faculty of Arts and Sciences, Near East University, Nicosia, Cyprus \\ ${ }^{4}$ Department of Dermatology and Venereology, Faculty of Medicine, Aksaray University, Niğde, Turkey
}

Adv Dermatol Allergol 2021; XXXVIII (3): 505-509

DOI: https://doi.org/10.5114/ada.2021.107937

\begin{abstract}
Introduction: Regular skin self-examination (SSE) is very important for the early diagnosis of malignant melanoma $(\mathrm{MM})$. Since sun exposure is the most important trigger factor for the development of skin cancers, effective and regular sun protection is the main preventive method.

Aim: To investigate the awareness of SSE, risky nevus and sun protection of the adolescents in the Turkish Republic of Northern Cyprus (TRNC).

Material and methods: The data used within this research were obtained from questionnaires administered to volunteer high school students in the TRNC.

Results: $39.8 \%$ of the participants included in the study stated that they conducted SSE. All the participants who said they conducted SSE reported that they performed a face exam. The body regions that the participants did not examine were the scalp (47.7\%), foot (36.9\%), back (35.4\%) and genital area (35.4\%). The features of the nevus perceived by the participants as risk factors included rapid growth, bleeding and itching. While $74 \%$ of respondents said they used sunscreen products, only $9 \%$ of users reported using them every day.

Conclusions: In the TRNC where the exposure to the sun is high, young people perform skin examinations at high rates in order to monitor their nevi. Nevertheless, the results of this research show that hard-to-reach areas are neglected.
\end{abstract}

Key words: nevus, melanoma, skin self-examination.

\section{Introduction}

While malignant melanoma (MM) is responsible for approximately $2 \%$ of skin cancers, it is the leading cause of skin cancer-related deaths [1]. Early diagnosis of MM can save lives and thus, awareness of this issue is extremely crucial [2]. With the increase in skin self-examination (SSE), the rate of dermatological consultations for suspicious nevus examination as well as the chance of early diagnosis has also seen an exponential rise. The rising global prevalence of MM emphasizes the importance of performing SSE through both, written and visual communication tools.

The main risk factors for MM development are: light skin type, natural or artificial ultraviolet rays, genetic predisposition (CDKN2A gene mutation), melanocytic nevus count, the presence of dysplastic nevus, occupational exposure, immune system suppression and history of melanoma, basal or squamous cell carcinoma [3, 4]. The transformation of melanocytes into tumour cells can occur in both genetically normal and predisposed individuals [5]. Data on the genetic susceptibility of the population to MM are non-existent.

Nevertheless, sun exposure, which is the most important triggering environmental factor in aetiology, is quite common amongst the young population. Skin cancers occur in more exposed areas of the body, such as the head, neck and hands. As a result, countries with high sunlight exposure have higher instances of MM [5]. Due to its geographical location, the island's yearly exposure to the sun is significantly high. Considering that Cyprus is located in

Address for correspondence: Didem Mullaaziz, Department of Dermatology and Venereology, Near East University, Faculty of Medicine, Near East Boulevard, 99138 Nicosia, North Cyprus Mersin 10, Turkey, phone: +90 6751000/1277, e-mail address: didem_mullaaziz@yahoo.com Received: 7.05.2020, accepted: 17.08.2020. 
the Eastern Mediterranean and receives an abundance of sunshine, the statistics of the Turkish Republic of Northern Cyprus (TRNC) for the last 25 years indicate that skin cancer has been the most common type of cancer [6]. For this reason, SSE should become a routine and compulsory application for young members of the population. A review of the existing literature has revealed that data regarding the use of SSE in Cyprus are not available.

\section{Aim}

This study aims to investigate the awareness of adolescents in the TRNC on the application of SSE, risky nevus and protection from the sun.

\section{Material and methods}

The data of this study were obtained from the questionnaire answers of high school students in the TRNC who voluntarily participated in the survey. The questionnaire was prepared by considering the literature review and included questions pertaining to the application of SSE and the use of sunscreens. The questionnaire included the following questions: demographic characteristics (i.e. age, gender), skin type (to determine the skin type, hair colour, skin colour, eye colour and skin's response to the sun were questioned), whether SSE was practiced; and if so, the last time it was performed (last month,

Table 1. Sociodemographic characteristics of the 163 participants

\begin{tabular}{lc}
\hline Variable & $\%$ \\
\hline Age [years]: & \\
\hline 14 & 2.5 \\
\hline 15 & 51.5 \\
\hline 16 & 36.8 \\
\hline 17 & 9.2 \\
\hline Gender: & 52.82 \\
\hline Female & 47.2 \\
\hline Male & \\
\hline Skin type: & 10.4 \\
\hline 2 & 47.9 \\
\hline 3 & 39.3 \\
\hline 4 & 2.5 \\
\hline 5 & 28.2 \\
\hline Nevus count: & 4 \\
\hline 0 & \\
\hline $1-2$ & 27 \\
\hline $3-10$ & \\
\hline 10 & \\
\hline
\end{tabular}

last 6 months, last year), for which body area (face, body, back, arm, leg, foot, hand, scalp, genital) and how often it was applied (every day, once a week, once a month, every 6 months, once a year); the total number of nevi on the participant's body $(0,1-2,3-10,>10)$, the skin cancer risk in the family, awareness on risky nevus (risky nevus characteristics predicted by the participants; dark colour, border irregularity, hairy, hard structure, colour variety, diameter, itching, bleeding, rapid growth, all of them, none of them), sunscreen usage and application (only sunny days, sometimes, every day).

\section{Statistical analysis}

Descriptive statistics were used when conducting data analysis, while SPSS 17 version software program and $\chi^{2}$ test were used for statistical analysis.

\section{Results}

In total, 163 (81.5\%) of the 200 participants involved completed and submitted the questionnaire in full. The ages of the participants in the study ranged between 14 and 17 and the mean age was calculated as $15.5 \pm 0.05$. In terms of gender distribution, there were 86 (52.8\%) females and 77 (47.2\%) males in the study. The majority of the participants had skin type 3 (47.9\%) and skin type 4 (39.3\%). When the participants were asked how many moles were present on their bodies, $27 \%$ stated that they had none, $40 \%$ claimed to have $1-2,28 \%$ mentioned they had 3-10, while 5\% said they had more than 10 nevi over their bodies (Table 1).

The main features that were identified by the participants as risk factors for nevi included: rapid growth, bleeding and pruritus (Figure 1).

$39.8 \%$ of the participants involved in the study stated that they practiced SSE. With $53.8 \%$ of SSE participants being female and $46.2 \%$ male, the most common skin types were identified as skin type 3 (47.7\%) and

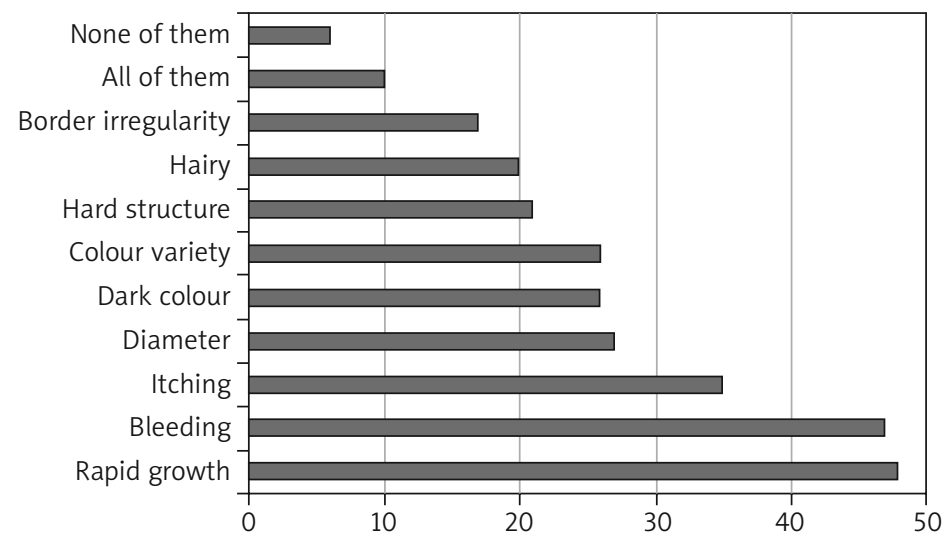

Figure 1. The distribution of risky properties foreseen by the participants in their nevi 
4 (36.9\%) for the particular group. There was no statistically significant difference between sex and skin type between the participants who practiced SSE and those who did not (Table 2).

Sixty percent of those who claimed to practice SSE mentioned that their most recent examination was conducted within the last month, $25 \%$ had received a consultation within the past 6 months and 15\% within the last year.

Body parts that were neglected by the participants when performing SSE saw higher rates of nevi. The body parts and corresponding percentages are as follows: over scalp (47.7\%), foot (36.9\%), back (35.4\%) and genitals (35.4\%). All participants stated that they performed facial examinations, albeit at different frequencies (Figure 2).

Among the participants who performed SSE, only one person reported a history of familial skin cancer.

While $74 \%$ of the participants stated that they used sunscreen products, it was noted that the percentage for females (62.5\%) was significantly higher than for males (37.5\%). When the frequency of sunscreen product usage was evaluated, $70 \%$ of the participants stated that they only used the product on sunny days, $21 \%$ said that they used it on some days, while only $9 \%$ reported that they used it every day. At least one nevus was reported by $75 \%$ of sunscreen users. However, no significant difference was detected between the rates of sunscreen use in patients with or without SSE application.

\section{Discussion}

Although MM usually develops from intact skin, it may also originate from pre-existing melanocytic nevi. The presence of more than one hundred melanocytic

Table 2. SSE according to sociodemographic factors among 65 students

\begin{tabular}{lc}
\hline Sociodemographic factors & \% Practicing SSE \\
\hline Overall & 39.8 \\
\hline Gender: & \\
\hline Female & 53.8 \\
\hline Male & 46.2 \\
\hline Skin type: & \\
\hline 2 & 12.3 \\
\hline 3 & 47.7 \\
\hline 4 & 36.9 \\
\hline 5 & 3.1 \\
\hline Nevus count: & \\
\hline 0 & 18.5 \\
\hline $1-2$ & 35.4 \\
\hline $3-10$ & 36.9 \\
\hline$>1$ & 9.2 \\
\hline
\end{tabular}

nevi is thought to increase the risk of MM 3-10 times [7]. Approximately $25 \%$ of melanoma cases have one or more atypical nevi, and $27 \%$ have more than 50 simple melanocytic nevi. The presence of more than one atypical nevi and the total number of simple melanocytic nevi lead to a relative risk increase for MM [5]. It is crucial for patients with risky nevi to have periodic dermatologic follow-ups. Additionally, it is especially important that these patients be instructed on how to perform SSE [8].

Although MM patients can detect suspicious lesions themselves, their general lack of awareness on the topic of MM may cause them to consult a dermatologist too late. One study reported that in $25 \%$ of the patients, the time between the detection of a suspicious lesion and the initiation of treatment took more than 1 year [9].

Routine SSE practice has the potential to increase the chance of early diagnosis and may even be the key to treatment and survival from melanoma [10]. Many organisations, including the American Academy of Dermatology, emphasize the importance of SSE and provide visual training materials for correct implementation [11]. It is estimated that SSE may reduce MM mortality by $63 \%$ [12]. In one study, it was reported that the sensitivity of the SSE ranged between $25 \%$ and $93 \%$ and the specificity was higher than 83-97\% [10]. Robinson et al. reported that the performance of SSE is directly related to the perceived risk associated with the development of melanoma or skin cancer on a person [12].

A dedicated web-based questionnaire was designed, and a cross-sectional study was conducted among readers of a Polish scientific portal, in which $60 \%$ of the participants reported that they practiced SSE [13]. However,

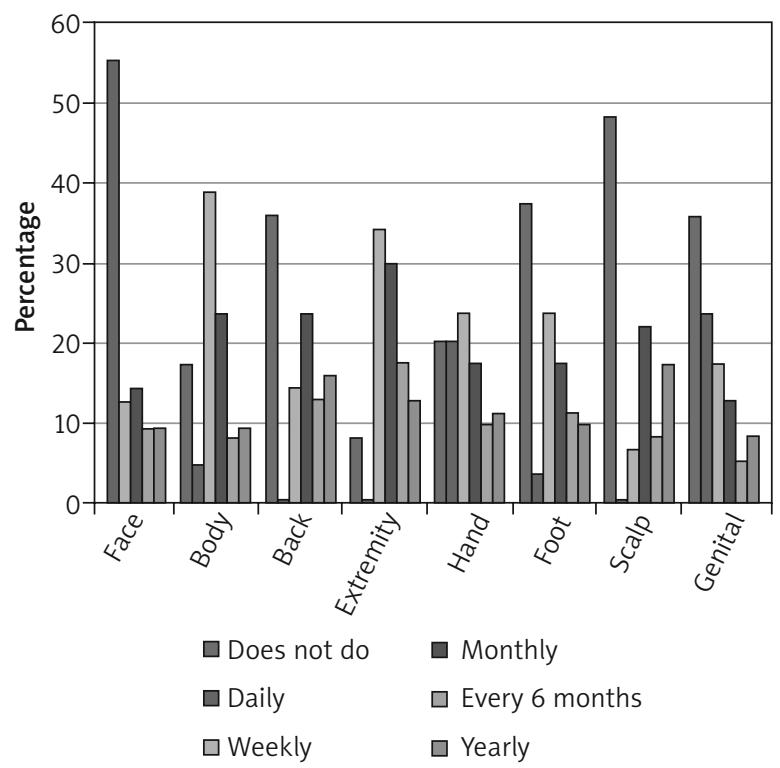

Figure 2. Body areas examined by SSE population and distribution of frequency of examinations 
in our study, a lower rate of SSE (39.8\%) implementation was detected.

MM carries a familial characteristic of $8-12 \%$ [14]. CDKN2A, a tumour suppressor, and melanocortin-1 gene defects have been shown to cause genetic predisposition to MM $[14,15]$. Dermatological examination of firstdegree relatives of patients diagnosed with melanoma is very important for early diagnosis. It should be noted that familial melanomas may occur at an earlier age [8]. In a survey study investigating the frequency of screening among children of parents with a family history of melanoma, $28 \%$ of parents reported that children had received a skin exam by a healthcare provider in the past 6 months [16]. In our study, only one person reported a history of familial skin cancer and admitted conducting SSE. In our country, no studies have been conducted on the level of awareness of this issue.

Melanin production in early childhood is very limited. For this reason, children are more susceptible to the harmful effects of sunlight. Studies show the importance of sun protection methods that are extremely effective in preventing the development of skin cancer, especially in the period up to the age of twenty [17]. Therefore, acquiring the habit of sun protection in childhood is of importance. In our study, some participants (74\%) stated that they used a high percentage of sunscreen products, while the majority (70\%) stated that they only used them on sunny days. Kyle et al. reported the same rate of sunscreen usage in Scottish adolescents (74\%) as in our study [18]. In this study, even though it was determined that high amounts of sunscreen were used, it was not used regularly.

The findings of our study show that participants who stated they used SSE methods, examined some body parts to a lesser extent. We concluded that the highest percentage of participants neglected to examine the scalp (47.7\%), foot (36.9\%), back (35.4\%) and genital area (35.4\%). This could be due to the difficulty in conducting self-examinations on such areas. Since the face is a body area that is considered particularly important by the adolescents in our study group, all participants who reported that they conducted SSE examined their faces at least once. Consequently, it is seen that the patients need to be informed about how and where to use SSE.

When the participants were questioned about what they considered to be the risk factors for nevi, their responses included rapid growth, bleeding, itching, diameter, dark colour, variety of colours, hard structure, and the presence of hair and border irregularities. These findings reveal that the participants care more about bleeding and pruritus, colour diversity and border disorder. These results suggest that people should be informed about the changes they should pay attention to when performing SSE and the stimuli that should be perceived as risk factors.

The limitations of this study include the insufficiency of the number of participants, the participants' knowl- edge about the SSE method, the accuracy and adequacy of the specific method of SSE they applied and our lack of information on how they had learned the SSE method.

\section{Conclusions}

In countries where sun exposure is quite high, such as Cyprus, it is important to use the SSE method to at least detect suspicious nevi visually and to consult a dermatologist for the early diagnosis of MM. Although warnings about examinations appear to have been effective, further studies should also focus on the correct way of conducting SSE. According to these findings, in our country, it is understood that detailed information is needed about the technique used to apply SSE, which body regions should be controlled, who is in the risk group and that it should be done more carefully. Since the SSE method is a very important step in early diagnosis, ensuring that it is performed routinely and correctly is a significant preventive health service and should be considered important for public health. Doctors in all branches are required to correctly guide their patients, and dermatologists should educate each patient in detail in this regard. Providing hands-on training sessions, including families, doctors and teachers, within the scope of health education in schools can help us achieve a complete solution. Our study is the first to be conducted in the TRNC on SSE and sun protection; therefore, it is clear that more comprehensive studies are needed in this regard.

\section{Conflict of interest}

The authors declare no conflict of interest.

\section{References}

1. Siegel R, Ma J, Zou Z, Jemal A. Cancer statistics. CA Cancer J Clin 2014; 64: 9-29.

2. Aitken JF, Janda M, Lowe JB, et al. Prevalence of whole-body skin self-examination in a population at high risk for skin cancer (Australia). Cancer Causes Control 2004; 15: 453-63.

3. Watts CG, Dieng M, Morton RL, et al. Clinical practice guidelines for identification, screening and follow-up of individuals at high risk of primary cutaneous melanoma: a systematic review. Br J Dermatol 2015; 172: 33-47.

4. Torrens R, Swan BA. Promoting prevention and early recognition of malignant melanoma. Dermatol Nurs 2009; 21: $115-22$.

5. Tuong W, Cheng LS, Armstrong AW. Melanoma: epidemiology, diagnosis, treatment, and outcomes. Dermatol Clin 2012; 30: 113-24.

6. Kaptanoğlu AF, Dalkan C, Hıncal E. Sun protection in the north Cyprus Turkish population: knowledge, attitude and behaviors of elementary school children and their families. Turkderm 2012; 46: 121-9.

7. Psaty EL, Scope A, Halpern AC, Marghoob AA. Defining the patient at high risk for melanoma. Int I Dermatol 2010; 49: 362-76.

8. Baykal C, Ekinci AP. Malign melanoma: risk factors and major clinical findings. Turk J Dermatol 2015; 9: 1-7. 
9. Blum A, Brand CU, Ellwanger U, et al. Awareness and early detection of cutaneous melanoma: an analysis of factors related to delay in treatment. Br J Dermatol 1999; 141: 783-7.

10. Hamidi R, Peng D, Cockburn M. Efficacy of skin self-examination for the early detection of melanoma. Int I Dermatol 2010; 49: 126-34.

11. Miller DR, Geller AC, Wyatt SW, et al. Melanoma awareness and self-examination practices: results of a United States survey. J Am Acad Dermatol 1996; 34: 962-70.

12. Robinson JK, Rigel DS, Amonette RA. What promotes skin self-examination? J Am Acad Dermatol 1998; 38: 752-7.

13. Kamińska-Winciorek G, Gajda M, Wydmański J, Tukiendorf A. What do Web users know about skin self-examination and melanoma symptoms? Asian Pac J Cancer Prev 2015; 16: 3051-6.

14. High WA, Robinson WA. Genetic mutations involved in melanoma: a summary of our current understanding. Adv Dermatol 2007; 23: 61-79.

15. Mayer JE, Swetter SM, Fu T, Geller AC. Screening, early detection, education, and trends for melanoma: current status (2007-2013) and future directions: Part I. Epidemiology, highrisk groups, clinical strategies, and diagnostic technology. J Am Acad Dermatol 2014; 71: 599.e1-599.e12.

16. Parsons BG, Hay JL, Aspinwall LG, et al. Understanding skin screening practices among children at elevated risk for melanoma to inform interventions for melanoma prevention and control. J Cancer Educ 2020; 35: 509-14.

17. Davis KJ, Cokkinides VE, Weinstock MA, et al. Summer sunburn and sun exposure among US youths ages 11 to 18: national prevalence and associated factors. Pediatrics 2002; 110: 27-35.

18. Kyle RG, Macmillan I, Forbat L, et al. Scottish adolescents' sun-related behaviours, tanning attitudes and associations with skin cancer awareness: a cross-sectional study. BMJ Open 2014; 4: e005137. 